\title{
Clinicopathological characteristics and treatment outcomes of occult breast cancer: a SEER population-based study
}

This article was published in the following Dove Press journal:

Cancer Management and Research

\author{
Li-Ping Ge ${ }^{1,2, *}$ \\ Xi-Yu Liu',2,* \\ Yi Xiao ${ }^{1,2}$ \\ Zong-Chao Gou ${ }^{1,2}$ \\ Shen Zhao ${ }^{1,2}$ \\ Yi-Zhou Jiang ${ }^{1,2}$ \\ Gen-Hong $\mathrm{Di}^{1,2}$ \\ 'Department of Breast Surgery, \\ Cancer Institute, Fudan University \\ Shanghai Cancer Center, Shanghai, \\ People's Republic of China; \\ ${ }^{2}$ Department of Oncology, Shanghai \\ Medical College, Fudan University, \\ Shanghai, People's Republic of China \\ *These authors contributed equally to \\ this work
}

Correspondence: Yi-Zhou Jiang;

Gen-Hong Di

Department of Breast Surgery, Cancer Institute, Fudan University Shanghai Cancer Center, 270 Dong-An Road, Shanghai 200032, People's Republic of China

Tel +862164175590

Fax +8621 64434556

Email yizhoujiang@fudan.edu.cn; dgh_2015@I26.com
Background: Occult breast cancer (OBC) is a rare type of breast cancer that has not been well studied. The clinicopathological characteristics and treatment recommendations for $\mathrm{OBC}$ are based on a limited number of retrospective studies and thus remain controversial.

Patients and methods: We identified 479 OBC patients and 115,739 non-OBC patients from 2004 to 2014 in and the Surveillance, Epidemiology, and End Results (SEER) database. The clinicopathological characteristics and survival outcomes were compared between $\mathrm{OBC}$ and non-OBC patients. We used the propensity score 1:1 matching analysis to evaluate $\mathrm{OBC}$ vs non-OBC comparison using balanced groups with respect to the observed covariates. We further divided the OBC population into four groups based on different treatment strategies. Univariable and multivariable analyses were used to calculate and compare the four treatment outcomes within the OBC population.

Results: OBC patients were older, exhibited a more advanced stage, a higher rate of negative estrogen receptor and progesterone receptor status, a higher rate of HER2-positive status, and a higher rate of $\geq 10$ positive lymph nodes, and were less likely to undergo surgical treatment than non-OBC patients. After adjustments for clinicopathological factors, the OBC patients exhibited a significantly better survival than the non-OBC patients $(P<0.001)$. This result was confirmed in a 1:1 matched case-control analysis. Within the four OBC treatment groups, we observed no difference in survival among the mastectomy group, the breast-conserving surgery (BCS) group, and the axillary lymph node dissection (ALND)-only group. The multivariable analysis revealed that the sentinel lymph node dissection-only group had the worst prognosis $(P<0.001)$. Conclusion: $\mathrm{OBC}$ has unique clinicopathological characteristics and a favorable prognosis compared with non-OBC. BCS plus ALND and radiotherapy showed a survival benefit that was similar to that of mastectomy for OBC patients.

Keywords: occult breast cancer, clinicopathological characteristics, treatment outcomes, SEER database

\section{Introduction}

Occult breast cancer $(\mathrm{OBC})$, which manifests as axillary lymph node (LN) metastasis without the evidence of a primary breast tumor on clinical examination or mammography, accounts for $0.3 \%-1.0 \%$ of all breast cancers. ${ }^{1-3}$ Although OBC has been described as having a natural history and biological behavior similar to that of node-positive non-OBC, ${ }^{4,5}$ the clinicopathological characteristics of $\mathrm{OBC}$, such as the hormone status, the HER2 status, and the LN involvement, are still unclear due to the rarity of this type of cancer. ${ }^{6-8}$ Furthermore, the survival outcomes of patients with $\mathrm{OBC}$ are still controversial. The 10-year overall survival (OS) rate varies from $47.5 \%$ 
to $67.1 \%{ }^{7} \mathrm{OBC}$ has been reported to show an outcome that was similar to or more favorable than that of stage II-III, $\mathrm{T} 1 \mathrm{~N} 1$, or small invasive breast cancers (pT1), ${ }^{5,6,9-11}$ but several studies reported an opposite conclusion. ${ }^{12,13}$

$\mathrm{OBC}$, which is extremely uncommon, continues to present a therapeutic challenge. Previous studies suggested that breast-conserving surgery (BCS) with axillary LN dissection (ALND) plus radiotherapy (RT) resulted in a survival outcome similar to that of mastectomy plus ALND. ${ }^{6,8}$ However, the current National Comprehensive Cancer Network (NCCN) guidelines recommend that patients with negative MRI results should be treated with mastectomy plus ALND or ALND plus whole breast irradiation. ${ }^{14}$ On the contrary, systemic chemotherapy, endocrine therapy, or trastuzumab is given for stage II or III disease according to the recommendations. ${ }^{14}$ However, the limited number of patients included in previous retrospective studies did not give us sufficient information to determine reliable treatment recommendations. ${ }^{15-17}$ Furthermore, updated cohort information is needed to further our understanding of $\mathrm{OBC}$ and to guide oncologists in treatment selection.

Utilizing the Surveillance, Epidemiology, and End Results (SEER) database, we sought to obtain the most recent population-based data to 1) study the clinicopathological characteristics and survival outcomes of $\mathrm{OBC}$ and 2) evaluate different treatment outcomes in $\mathrm{OBC}$ patients.

\section{Patients and methods}

\section{Ethics statement}

Our study was approved by the independent ethical committee/institutional review board at Fudan University Shanghai Cancer Center (Shanghai Cancer Center Ethical Committee). The data released by the SEER database are publicly available and deidentified and thus do not require patient informed consent.

\section{Patient selection and data acquisition}

We used SEER data released in November 2016, which include data from 18 population-based cancer registries (1973-2014) and cover approximately $28 \%$ of US cancer patients. The inclusion criteria used to identify eligible patients were as follows: females older than 18 years of age, breast-adjusted American Joint Committee on Cancer (AJCC) sixth edition (1988+) stage T0-3N1-3M0, one or more positive LNs, unilateral breast cancer, breast cancer (ICD-O-3 site code C50) as the first and only cancer diagnosis, either no surgical treatment or surgical treatment with BCS or mastectomy, diagnosis not obtained from a death certificate or autopsy, only one primary site, a known number of examined LNs, and a known time of diagnosis between January 1, 2004, and December 31, 2014. In all, 116,218 patients were included. Of these patients, 479 (stage T0N1-3M0) were diagnosed with OBC and 115,739 (stage T1-3N1-3M0) were diagnosed with non-OBC.

An analysis of the clinicopathological characteristics of $\mathrm{OBC}$ and non-OBC included the year of diagnosis, age at diagnosis, race, marital status, laterality, grade, AJCC stage, AJCC N stage, estrogen receptor (ER) status, progesterone receptor (PR) status, HER2 status, breast cancer subtype, breast surgery type, RT, number of examined LNs, and number of positive LNs. We considered the year of diagnosis as a binary variable classified into the following two groups: 2004-2009 and 2010-2014. The age at diagnosis was also considered a binary variable and was classified into the following two groups: 18-49 years and more than 50 years. For HER2 status, data were available only after 2010 due to limitations in the SEER database. Moreover, the number of examined LNs was classified into the following three groups: $1-3,4-9$, and $\geq 10$, while the number of positive LNs was categorized into the following four groups: $1-3,4-9, \geq 10$, and unknown.

\section{Treatment course}

Patients who underwent breast surgery (BCS, mastectomy, or no surgery), RT, sentinel LN dissection (SLND) and/or ALND were identified based on the SEER variables. The SEER database did not specify the type of axillary LN surgery performed; therefore, surrogate data were used to categorize patients as having undergone SLND or ALND. Patients in whom one to five LNs were examined were considered to have undergone SLND alone, whereas patients in whom more than five LNs were examined were considered to have undergone ALND. These definitions were based on the AJCC definition of a standard minimum ALND (at least six LNs). ${ }^{18}$ The treatment groups of OBC population were defined according to breast surgery, LN status, and RT: 1) the mastectomy group (mastectomy plus ALND, with or without RT); 2) the BCS group (BCS plus both ALND and RT); 3) the ALND-only group; and 4) the SLND-only group.

\section{Outcome measurement}

In this study, breast cancer-specific survival (BCSS), which we used as the primary study outcome, was calculated from the date of diagnosis to the date of death from breast cancer. Patients who died from other causes unrelated to their breast cancer diagnosis and those who remained alive were cen- 
sored on the date of death or the date of last contact. OS, the secondary outcome, was measured from the date of diagnosis to the date of death from any cause, and patients who were alive on the date of final follow-up were censored. The cutoff date for the study was predetermined by the submission databases; the SEER November 2016 submission databases contain complete death data through 2014. Therefore, December 31, 2014, was assigned as the cutoff date for the study.

\section{Statistical analyses}

The clinicopathological characteristics were compared across groups using the chi-squared test or Fisher's exact test. Kaplan-Meier curves were used to calculate the 5-year BCSS and OS rates, and the log-rank test was used to determine differences between curves. Univariable and multivariable Cox proportional hazards regression models were applied to identify factors associated with survival; the HR and 95\% CI were reported. To account for large sample size, we selected the variables with $P<0.001$ (Table 1) or $P<0.05$ (Table 2) which were significantly associated with the BCSS or OS in the univariable analysis. To account for differences in the baseline characteristics across the two breast cancer subtype groups, we matched each OBC patient to one non-OBC patient according to the following predetermined factors: age, year of diagnosis, race, grade, AJCC N stage, ER status, PR status, type of breast surgery, and use of RT. Since 1:1 matching is the most common implementation of propensity score matching and the statistical power does not decrease, ${ }^{19}$ we used psmatch2 to perform a "one-to-one match" with the caliper width set as 0.05 in Stata statistical software for propensity score matching. Furthermore, patients in a matched pair have the same value of the propensity score. The stratified Cox model was conducted within matched pairs. Survival curves were estimated by the Kaplan-Meier method, and a stratified log-rank test was used to compare survival curves after propensity score matching. All statistical analyses were performed using Stata statistical software, version 13.0 (StataCorp LP, College Station, TX, USA). A two-tailed $P<0.05$ was considered statistically significant.

\section{Results \\ Clinicopathological characteristics of $\mathrm{OBC}$ patients}

The clinicopathological characteristics of all 116,218 selected patients are summarized in Table 3. Compared with non-OBC patients, $\mathrm{OBC}$ patients presented a higher proportion of stage III disease $(39.87 \%$ vs $33.14 \%, P=0.002)$, N3 classifica- tion $(19.62 \%$ vs $9.06 \%, P<0.001)$, ER negativity $(36.54 \%$ vs $19.37 \%, P<0.001)$ and PR-negative status $(52.19 \%$ vs $29.74 \%, P<0.001)$, and HER2-positive status $(12.11 \%$ vs $7.87 \%, P<0.001)$. Furthermore, the proportion of patients with $\geq 10$ examined LNs, $\geq 10$ positive LNs, and no surgery was significantly higher in the $\mathrm{OBC}$ group than in the non-OBC group $(68.06 \%$ vs $62.13 \%, P=0.001 ; 15.87 \%$ vs $8.37 \%, P<0.001 ; 48.85 \%$ vs $0.87 \%, P<0.001$, respectively). RT, however, was performed with similar frequency in both groups. These data indicated that the baseline characteristics of $\mathrm{OBC}$ are distinct from those of non-OBC.

\section{Comparison of survival between OBC and non-OBC patients}

After the baseline characteristics were summarized, we further evaluated BCSS and OS in these two groups using the Kaplan-Meier survival curves (Figure S1). The 5-year BCSS rate of the OBC group was 88.02\% (95\% CI 84.06-91.04\%), and the 5-year OS rate of the OBC group was $85.51 \%(95 \%$ CI 81.27-88.85\%), which were similar to the rates observed in the non-OBC group. We used the Cox proportional hazards model to investigate the effect of baseline characteristics on survival outcomes. A univariable analysis revealed that the year of diagnosis, age, race, marital status, tumor grade, ER status, PR status, HER2 status, breast surgery type, RT status, and the number of positive LNs were significantly associated with the BCSS and OS $(P<0.001)$. However, the difference between the $\mathrm{OBC}$ group and the non-OBC group was not statistically significant (Table S1). Furthermore, we included all variables mentioned earlier in the multivariable analysis (Table 1). However, after adjustment for potential confounders, $\mathrm{OBC}$ was identified as an independent protective factor for both BCSS (HR $=0.37,95 \%$ CI $0.27-0.50$, $P<0.001)$ and $\mathrm{OS}(\mathrm{HR}=0.41,95 \%$ CI $0.31-0.52, P<0.001)$.

\section{Survival analysis in matched groups}

To further evaluate the detected differences between $\mathrm{OBC}$ and non-OBC groups, we performed a 1:1 (OBC:non-OBC) matched case-control analysis using the propensity score matching method. We defined a group of 794 patients, where 397 patients were included in each group (Table S2). For the matched groups, we found no statistically significant difference in the baseline characteristics between the $\mathrm{OBC}$ patients and the non-OBC patients. As expected, the OBC patients demonstrated a better prognosis in terms of both BCSS and OS (Figure 1; $P<0.001$ and $P<0.001$, respectively), which was consistent with the stratified Cox models 
Table I Multivariable analysis of BCSS and OS in all patients

\begin{tabular}{|c|c|c|c|c|c|c|}
\hline \multirow[t]{2}{*}{ Variables } & \multicolumn{3}{|l|}{ BCSS } & \multicolumn{3}{|l|}{ OS } \\
\hline & $\bar{n}$ & HR (95\% Cl) & $P$-value & $n$ & HR (95\% Cl) & $P$-value \\
\hline Group & & & $<0.001$ & & & $<0.001$ \\
\hline OBC & 51 & $0.37(0.27-0.50)$ & $<0.001$ & 68 & $0.4 \mathrm{I}(0.3 \mathrm{I}-0.52)$ & $<0.001$ \\
\hline non-OBC & 12,847 & Reference & - & 18,644 & Reference & - \\
\hline Year of diagnosis & & & 0.402 & & & 0.831 \\
\hline 2004-2009 & 10,260 & Reference & - & 15,020 & Reference & - \\
\hline 20I0-2014 & 2,638 & $0.93(0.77-1.11)$ & 0.402 & 3,692 & $1.02(0.88-1.17)$ & 0.831 \\
\hline Age at diagnosis (years) & & & $<0.001$ & & & $<0.001$ \\
\hline $18-49$ & 3,877 & Reference & - & 4,340 & Reference & - \\
\hline$\geq 50$ & 9,021 & $1.25(1.20-1.30)$ & $<0.001$ & 14,372 & $1.72(1.66-1.78)$ & $<0.001$ \\
\hline Race & & & $<0.001$ & & & $<0.001$ \\
\hline White & 9,730 & Reference & - & 14,356 & Reference & - \\
\hline Black & 2,294 & $1.31(1.25-1.37)$ & $<0.001$ & 3,153 & $1.25(1.20-1.30)$ & $<0.001$ \\
\hline Others $^{\mathrm{a}}$ & 848 & $0.79(0.73-0.84)$ & $<0.001$ & 1,167 & $0.77(0.72-0.82)$ & $<0.001$ \\
\hline Unknown & 26 & $0.48(0.33-0.70)$ & $<0.001$ & 36 & $0.43(0.3 \mathrm{I}-0.6 \mathrm{I})$ & $<0.001$ \\
\hline Marital status & & & $<0.001$ & & & $<0.001$ \\
\hline Married & 6,592 & Reference & - & 8,663 & Reference & - \\
\hline Not married ${ }^{b}$ & 5,875 & $1.32(1.27-1.36)$ & $<0.001$ & 9,407 & $1.58(1.54-1.63)$ & $<0.001$ \\
\hline Unknown & 431 & $1.13(1.02-1.25)$ & 0.014 & 642 & $1.26(1.16-1.36)$ & $<0.001$ \\
\hline Grade & & & $<0.001$ & & & $<0.001$ \\
\hline 1 and 2 & 4,211 & Reference & - & 7,482 & Reference & - \\
\hline 3 and UD & 8,220 & $1.78(1.71-1.85)$ & $<0.001$ & 1,0529 & $1.45(1.40-1.50)$ & $<0.001$ \\
\hline Unknown & 467 & $1.13(1.03-1.25)$ & 0.012 & 701 & $1.18(1.09-1.27)$ & $<0.001$ \\
\hline ER status & & & $<0.001$ & & & $<0.001$ \\
\hline Positive & 7,308 & Reference & - & $|1,69|$ & Reference & - \\
\hline Negative & 5,057 & $1.52(1.44-1.60)$ & $<0.001$ & 6,181 & $1.40(1.34-1.46)$ & $<0.001$ \\
\hline Unknownc & 533 & $1.25(1.06-1.47)$ & 0.009 & 840 & $1.23(1.07-1.40)$ & 0.004 \\
\hline PR status & & & $<0.001$ & & & $<0.001$ \\
\hline Positive & 5,530 & Reference & - & 9,086 & Reference & - \\
\hline Negative & 6,708 & $1.63(1.55-1.72)$ & $<0.001$ & 8,582 & $1.44(1.38-1.50)$ & $<0.001$ \\
\hline Unknownc & 660 & $1.29(1.11-1.5 I)$ & $<0.001$ & $\mathrm{I}, 044$ & I.2I (I.07-I.37) & $<0.001$ \\
\hline HER2 status & & & $<0.001$ & & & $<0.001$ \\
\hline Positive & 395 & Reference & - & 523 & Reference & - \\
\hline Negative & 2,119 & $1.76(1.58-1.96)$ & $<0.001$ & 2,962 & I.63 (I.49-I.79) & $<0.001$ \\
\hline Unknown & 10,384 & $1.48(1.21-1.82)$ & $<0.001$ & 15,227 & $1.60(1.36-1.88)$ & $<0.001$ \\
\hline Breast surgery type & & & $<0.001$ & & & $<0.001$ \\
\hline Mastectomy & 8,803 & Reference & - & 5,892 & Reference & - \\
\hline BCS & 3,894 & $0.72(0.69-0.75)$ & $<0.001$ & 12,563 & $0.74(0.72-0.77)$ & $<0.001$ \\
\hline No surgery & 201 & $2.17(0.69-1.36)$ & $<0.001$ & 257 & $1.80(1.58-2.06)$ & $<0.001$ \\
\hline RT & & & $<0.001$ & & & $<0.001$ \\
\hline Yes & 6,486 & Reference & - & 8,604 & Reference & - \\
\hline No & 6,412 & $1.23(1.19-1.27)$ & $<0.001$ & 10,108 & $1.39(1.35-1.43)$ & $<0.001$ \\
\hline No. of positive LNs & & & $<0.001$ & & & $<0.001$ \\
\hline $1-3$ & 6,254 & Reference & - & 10,244 & Reference & - \\
\hline $4-9$ & 3,802 & $2.13(2.04-2.22)$ & $<0.001$ & 5,054 & $1.79(1.73-1.86)$ & $<0.001$ \\
\hline$\geq 10$ & 2,808 & $3.79(3.62-3.97)$ & $<0.001$ & 3,376 & $2.92(2.8 \mathrm{I}-3.04)$ & $<0.001$ \\
\hline Unknown & 34 & $0.97(0.69-1.36)$ & 0.843 & 38 & $0.80(0.58-I .1 \mathrm{I})$ & 0.179 \\
\hline
\end{tabular}

Notes: ancluding American Indian/Alaskan native and Asian/Pacific Islander. 'Including divorced, separated, single (never married), and widowed. 'Including borderline and unknown. ${ }^{d H E R 2+~ u n k n o w n ~ i n c l u d e s ~ b o r d e r l i n e, ~ u n k n o w n, ~ a n d ~ b e f o r e ~} 2010$ breast cancer. Reference indicates the control population.

Abbreviations: BCS, breast-conserving surgery; BCSS, breast cancer-specific survival; ER, estrogen receptor; LNs, lymph nodes; n, the number of deaths; OBC, occult breast cancer; OS, overall survival; PR, progesterone receptor; RT, radiotherapy; UD, undifferentiated. 
Table 2 Multivariable analysis of BCSS and OS in OBC patients

\begin{tabular}{|c|c|c|c|c|c|c|}
\hline \multirow[t]{2}{*}{ Variables } & \multicolumn{3}{|c|}{ BCSS } & \multicolumn{3}{|c|}{ OS } \\
\hline & $n$ & HR (95\% CI) & $P$-value & $n$ & HR (95\% CI) & P-value \\
\hline \multicolumn{7}{|l|}{ ER status } \\
\hline Positive & 15 & Reference & - & 21 & Reference & - \\
\hline Negative & 21 & $\mathrm{I} .45(0.6 \mathrm{I}-3.47)$ & 0.403 & 30 & I.48 (0.72-3.05) & 0.292 \\
\hline Unknown ${ }^{\mathrm{a}}$ & 5 & $2.83(0.32-31.28)$ & 0.524 & 6 & $3.19(0.36-28.31)$ & 0.297 \\
\hline \multicolumn{7}{|l|}{ PR status } \\
\hline Positive & 10 & Reference & - & 14 & Reference & - \\
\hline Negative & 26 & $1.62(0.63-4.16)$ & 0.317 & 36 & $1.53(0.69-3.38)$ & 0.296 \\
\hline Unknown a & 5 & $0.74(0.29-6.27)$ & 0.795 & 7 & $0.63(0.08-5.00)$ & 0.660 \\
\hline \multicolumn{7}{|c|}{ Treatment strategy } \\
\hline Mastectomy group & 17 & Reference & - & 24 & Reference & - \\
\hline BCS group & 12 & $0.81(0.38-1.72)$ & 0.588 & 14 & $0.64(0.33-1.25)$ & 0.331 \\
\hline ALND-only group & 4 & $1.17(0.38-3.61)$ & 0.785 & 7 & $1.33(0.55-3.17)$ & 0.527 \\
\hline SLND-only group & 8 & $10.12(3.09-33.18)$ & $<0.001$ & 12 & $7.95(3.13-20.17)$ & $<0.001$ \\
\hline \multicolumn{7}{|c|}{ No. of positive LNs } \\
\hline $1-3$ & II & Reference & - & 18 & Reference & - \\
\hline 4-9 & 9 & $4.73(1.55-14.4 I)$ & 0.006 & 15 & $4.30(1.85-10.00)$ & 0.001 \\
\hline$\geq 10$ & 19 & II.9| (4.30-32.97) & $<0.001$ & 21 & $7.19(3.21-16.12)$ & $<0.001$ \\
\hline Unknown & 2 & $6.49(1.37-30.69)$ & 0.018 & 3 & $6.97(1.96-24.81)$ & 0.003 \\
\hline
\end{tabular}

Note: ancluding borderline and unknown.

Abbreviations: ALND, axillary LN dissection; BCS, breast-conserving surgery; BCSS, breast cancer-specific survival; ER, estrogen receptor; LNs, lymph nodes; $n$, the number of deaths; OBC, occult breast cancer; OS, overall survival; PR, progesterone receptor; SLND, sentinel LN dissection.

( Table S3 $;$ BCSS HR $=0.40,95 \%$ CI $0.27-0.50, P<0.001$; OS $\mathrm{HR}=0.41,95 \% \mathrm{CI} 0.31-0.52, P<0.001)$. In conclusion, OBC predicted a better prognosis than non-OBC after adjustment for clinicopathological characteristics.

\section{Clinical outcomes of OBC in different treatment groups}

Since local treatment options for OBC are a subject of intense interest, we further evaluated the relationship between the type of surgery and survival in OBC. We classified the OBC patients into four groups based on different treatment strategies as follows: $153(38.8 \%)$ patients were treated with mastectomy plus ALND, with or without RT; 151 (38.3\%) patients were treated with BCS plus both ALND and RT; 54 (13.7\%) patients received ALND only; and 36 patients (9.1\%) underwent SLND only. The clinicopathological characteristics of these four treatment groups are summarized in Table S4.

Kaplan-Meier survival curves were used to evaluate the clinical outcomes in these four treatment groups (Figure 2). Significant differences were observed in $\operatorname{BCSS}(P=0.023)$ and OS $(P<0.001)$ between the treatment groups. Since survival was also affected by ER status, PR status, and the number of positive LNs (Table S5), we performed a multivariable analysis to adjust for these variables (Table 2). After the adjustment, the SLND-only group significantly demonstrated the worst BCSS $(P<0.001)$ and OS $(P<0.001)$. No differences were detected in the survival rates among the mastectomy group, the BCS group and the ALND-only group in either BCSS or OS (Table S6).

\section{Discussion}

In this retrospective study, we investigated the clinicopathological characteristics and treatment outcomes of OBC based on a large population from the SEER database. Compared with non-OBC patients, OBC patients presented with distinct baseline characteristics and different BCSS and OS rates. After adjustment for baseline characteristics, OBC patients demonstrated a significant survival advantage over non-OBC patients. Further analyses that evaluated the treatment strategies of the $\mathrm{OBC}$ patients revealed that the mastectomy group, the BCS group, and the ALND-only group had comparable outcomes.

Our analysis took advantage of the SEER database to investigate the clinicopathological characteristics of the OBC cohort based on a large population size. Compared with non-OBC, this special type of breast cancer was associated with older age, a more advanced stage, a higher proportion of negative hormone receptor expression, a higher proportion of HER2-positive status, a greater likelihood of having $\geq 10$ positive LNs, and a lower likelihood of surgical treatment; some of these associations were concordant with the 
Table 3 Clinicopathological characteristics of patients with $O B C$ and non-OBC

\begin{tabular}{|c|c|c|c|}
\hline Variables & OBC $(\mathrm{N}=479),(\%)$ & non-OBC $(N=\mid 15,739),(\%)$ & $P$-value ${ }^{a}$ \\
\hline Year of diagnosis & & & 0.787 \\
\hline 2004-2009 & $258(53.86)$ & $61,624(53.24)$ & \\
\hline $2010-2014$ & $221(46.14)$ & $54,115(46.76)$ & \\
\hline Age at diagnosis (years) & & & $<0.001$ \\
\hline $18-49$ & II 3 (23.59) & $37,14 \mid$ (32.09) & \\
\hline$\geq 50$ & $366(76.4 I)$ & 78,598 (67.9I) & \\
\hline Race & & & 0.400 \\
\hline White & $388(81.00)$ & $90,859(78.5 \mathrm{I})$ & \\
\hline Black & $56(I 1.69)$ & $14,110(12.19)$ & \\
\hline Others ${ }^{b}$ & $34(7.10)$ & $10,152(8.77)$ & \\
\hline Unknown & $\mathrm{I}(0.2 \mathrm{I})$ & $61,8(0.53)$ & \\
\hline Marital status & & & 0.260 \\
\hline Married & $276(57.62)$ & 66,767 (57.69) & \\
\hline Not married ${ }^{c}$ & $191(39.87)$ & $44,443(38.40)$ & \\
\hline Unknown & $12(2.5 \mathrm{I})$ & $4,529(3.91)$ & \\
\hline Laterality & & & 0.001 \\
\hline Left & $246(51.36)$ & $58,563(50.60)$ & \\
\hline Right & $232(48.43)$ & 57,161 (49.39) & \\
\hline Only one side, NOS & $\mathrm{I}(0.2 \mathrm{I})$ & $15(0.01)$ & \\
\hline Grade & & & $<0.001$ \\
\hline 1 and 2 & $29(6.06)$ & $63,146(54.56)$ & \\
\hline 3 and UD & $113(23.59)$ & $48,818(42.18)$ & \\
\hline Unknown & $337(70.35)$ & $3,775(3.26)$ & \\
\hline AJCC stage & & & 0.002 \\
\hline II & $288(60.13)$ & $77,385(66.86)$ & \\
\hline III & $191(39.87)$ & $38,354(33.14)$ & \\
\hline AJCC $N$ stage & & & $<0.001$ \\
\hline NI & $288(60.13)$ & $84,314(72.85)$ & \\
\hline N2 & $97(20.25)$ & 20,940 (18.09) & \\
\hline N3 & $94(19.62)$ & $10,485(9.06)$ & \\
\hline ER status & & & $<0.001$ \\
\hline Positive & $265(55.32)$ & $90,126(77.87)$ & \\
\hline Negative & $175(36.54)$ & 22,415 (19.37) & \\
\hline Others $^{d}$ & $39(8.14)$ & $3,198(2.76)$ & \\
\hline PR status & & & $<0.001$ \\
\hline Positive & 179 (37.37) & $77,211(66.71)$ & \\
\hline Negative & $250(52.19)$ & 34,42 I (29.74) & \\
\hline Others $^{d}$ & $50(10.44)$ & $4,107(3.55)$ & \\
\hline HER2 status & & & $<0.001$ \\
\hline Positive & $58(12.11)$ & $9,113(7.87)$ & \\
\hline Negative & $136(28.39)$ & $4,260 I(36.8 I)$ & \\
\hline Others ${ }^{\mathrm{e}}$ & $285(59.50)$ & $6,4025(55.32)$ & \\
\hline Breast subtype & & & $<0.001$ \\
\hline HoR+/Her2+ & $38(7.93)$ & $6,408(5.54)$ & \\
\hline HoR+/Her2- & $92(19.21)$ & $36,839(31.83)$ & \\
\hline HoR-/Her2+ & $18(3.76)$ & $2,691(2.33)$ & \\
\hline Triple negative & $44(9.19)$ & $5,708(4.93)$ & \\
\hline Unknown & $287(59.91)$ & $64,093(55.37)$ & \\
\hline Breast surgery type & & & $<0.001$ \\
\hline $\mathrm{BCS}$ & $66(13.78)$ & $48,245(4 I .68)$ & \\
\hline Mastectomy & $179(37.37)$ & $66,487(57.45)$ & \\
\hline No surgery & $234(48.85)$ & $\mathrm{I}, 007(0.87)$ & \\
\hline RT & & & 0.294 \\
\hline Yes & $269(59.16)$ & $62,225(53.76)$ & \\
\hline No & $210(43.84)$ & $53,5 \mathrm{I} 4(46.24)$ & \\
\hline
\end{tabular}


Table 3 (Continued)

\begin{tabular}{llll}
\hline Variables & OBC (N=479), (\%) & non-OBC (N=I I5,739), (\%) & $P$-value ${ }^{\mathrm{a}}$ \\
\hline No. of examined LNs & $78(16.28)$ & & 0.001 \\
I-3 & $75(15.66)$ & $17,408(15.04)$ \\
$4-9$ & $326(68.06)$ & $26,42 I(22.83)$ \\
$\geq 10$ & & $71,910(62.13)$ \\
No. of positive LNs & $292(60.96)$ & \\
I-3 & $94(19.62)$ & $82,638(71.40)$ \\
$4-9$ & $76(15.87)$ & $22,557(19.49)$ \\
$\geq 10$ & $17(3.55)$ & $9,686(8.37)$ \\
Unknown & $858(0.74)$ & $<0.001$ \\
\hline
\end{tabular}

Notes: ${ }^{2}$-value was assessed using Pearson's chi-squared test or Fisher's exact test. 'Including American Indian/Alaskan native and Asian/Pacific Islander. 'Including divorced, separated, single (never married), and widowed. Including borderline and unknown. ${ }^{\text {eHER2 }}+$ unknown includes borderline, unknown, and before 2010 breast cancer. Abbreviations: AJCC, American Joint Committee on Cancer; BCS, breast-conserving surgery; ER, estrogen receptor; HoR, hormone receptor; LNs, lymph nodes; NOS, not otherwise specified; OBC, occult breast cancer; PR, progesterone receptor; RT, radiotherapy; UD, undifferentiated.

findings of previous studies..$^{5,10,11,20}$ The higher proportion of negative ER and PR status in OBC than in non-OBC might be explained by the discordance in this status between the primary tumor and LN metastases. ${ }^{21,22}$ Due to the absence of clinically, radiologically, or pathologically identifiable breast tumors, the receptor status in OBC patients was detected from the metastatic LNs. Discordance was reported to result from the following three main factors: false-positive and false-negative results in the assessment of receptor expression, the heterogeneity of receptor expression in the same tumor, and evolutionary modifications in the biology of the cancer. ${ }^{23-25}$ These baseline characteristics reveal that we cannot regard this rare disease in the same manner as palpable breast cancers.

Our study demonstrated that OBC patients showed higher survival rates than non-OBC patients. Furthermore, the 1:1 matching study confirmed this result. Collectively, these survival advantages suggest that $\mathrm{OBC}$ has a relatively benign biological behavior even though it initially presents as axillary LN metastasis, a concept that runs counter to our understanding of locally metastatic breast cancer. ${ }^{26,27}$ Pentheroudakis et $\mathrm{al}^{5}$ reported that $\mathrm{OBC}$ grew more indolent than overt breast cancer, which is an observation that adds evidence to our conclusion.

Treatment strategies for OBC have varied over the years, and to date, no definite consensus has been reached. Mastectomy with ALND, a regimen that was first described by Halsted, ${ }^{28}$ has traditionally been believed to provide the most effective local treatment for $\mathrm{OBC}$ patients. The advantage of mastectomy for $\mathrm{OBC}$ treatment is that it can confirm the diagnosis of $\mathrm{OBC}$ after a detailed pathological examination, ${ }^{29}$ despite that no primary tumor was found in $30 \%-60 \%$ of patients. ${ }^{1,30-32}$ In recent years, published studies have demonstrated that mastectomy did not improve survival outcomes compared with $\mathrm{BCS}$ in $\mathrm{OBC}$ patients and that BCS plus ALND with RT is an acceptable alternative. ${ }^{8,29,33-37}$ Consistent with these studies, we observed similar BCSS and OS outcomes in the BCS group and in the mastectomy group. He et $\mathrm{al}^{8}$ also observed similar locoregional recurrence-free survival (LRFS) and recurrence/ metastasis-free survival (RFS) outcomes between these two treatment groups in a retrospective study that assessed patients from 1998 to 2010. Our study provides additional evidence that BCS plus both ALND and RT instead of mastectomy with or without RT might be a favorable management strategy for OBC patients. However, in this study, the ALND-only group exhibited BCSS and OS rates similar to those of the BCS group and the mastectomy group, a result that was different from the

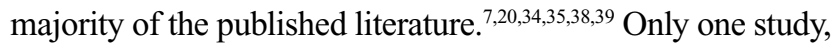
conducted in Korea, supported our finding, with the explanation that the ALND-only group in their study population had a high prevalence of stage N3 disease. ${ }^{6}$ In our study, potential explanations for this observation are that the ALND-only group included a limited number of patients $(n=54)$ and had a higher proportion of patients with $1-3$ positive LNs $(57.41 \%$, $P=0.003)$. In addition, defining ALND by different criteria may affect the survival outcomes. Other SEER database analyses categorized more than three examined LNs as having undergone ALND; ${ }^{7,40}$ this definition was inconsistent with our definition of ALND as the examination of more than five LNs. Therefore, further clinical studies are needed to evaluate this controversial conclusion and to confirm that ALND is an effective standalone treatment strategy for $\mathrm{OBC}$ patients. Moreover, our analysis found that the SLND-only group had the worst survival rate, which suggests that the extent of LN examination and the type of locoregional therapy are important for OBC patients. ${ }^{7,20,41}$

The advantage of this study is that it is based on a database that to the best of our knowledge includes the largest patient population in the world. Inevitably, the current study still has several limitations. First, this study was a retrospective cohort study and might have some potential selection biases and 
A

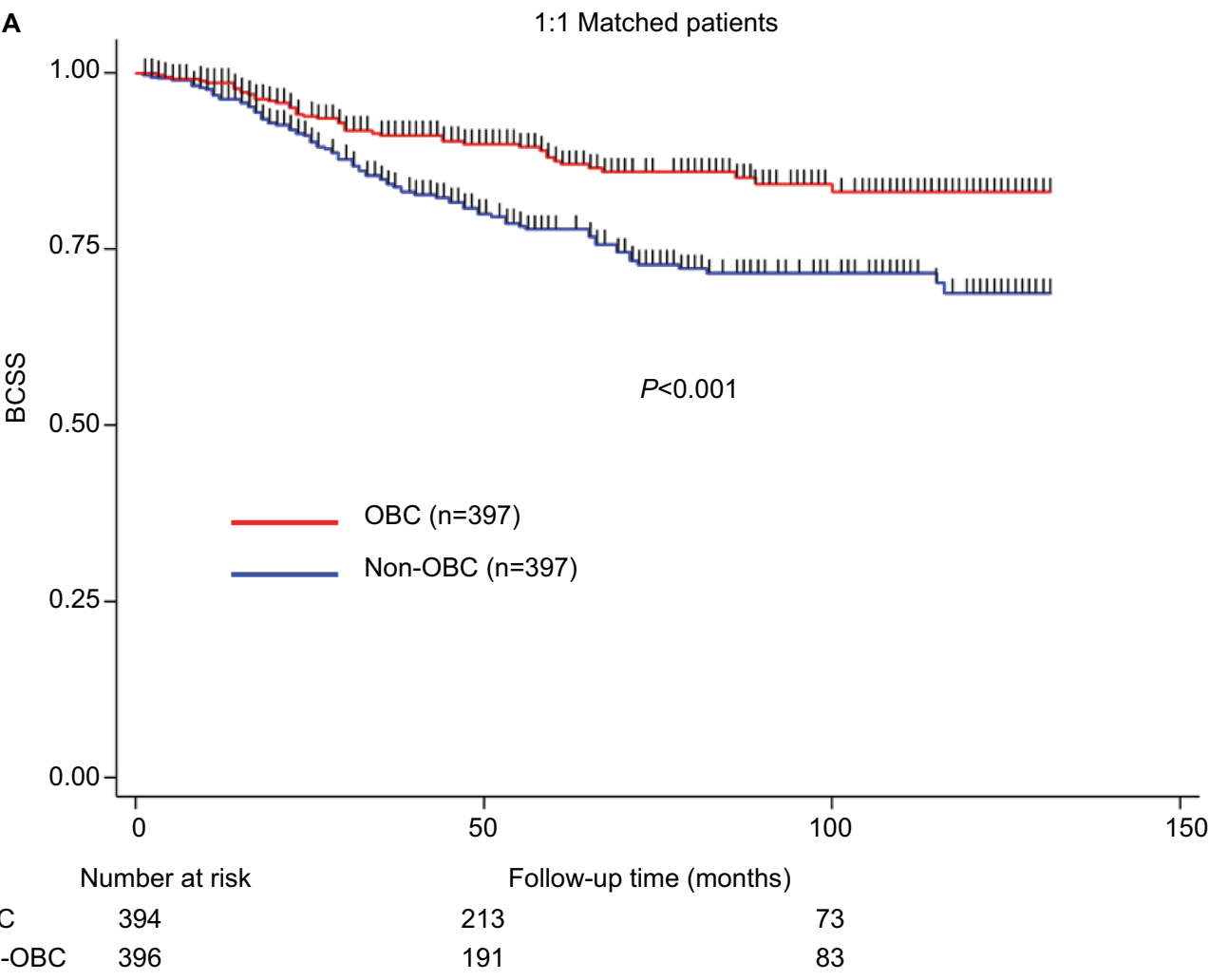

B

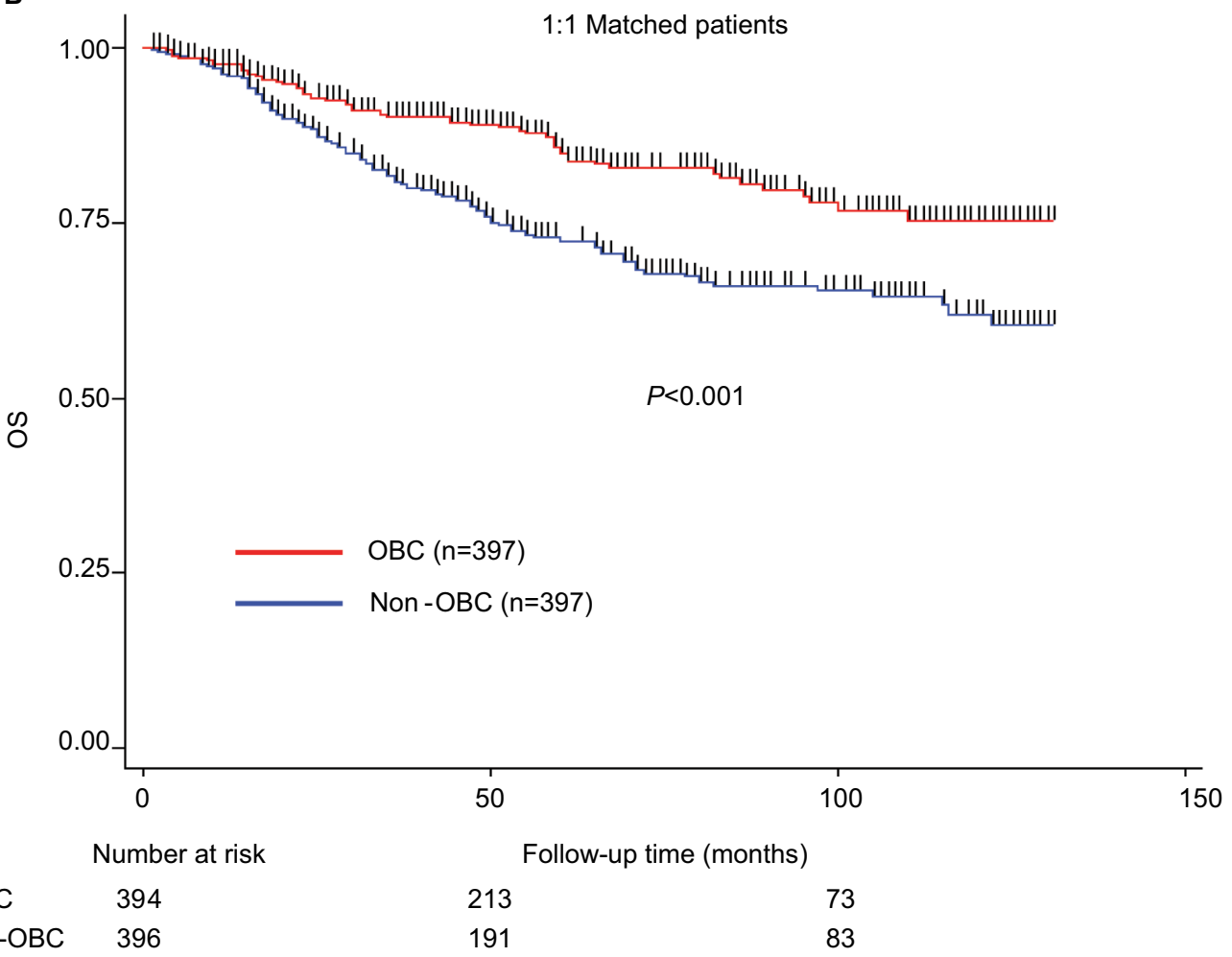

Figure I Kaplan-Meier survival curves according to the two groups of I:I matched patients.

Notes: (A) BCSS. (B) OS. Stratified log-rank tests were compared between OBC and non-OBC patients.

Abbreviations: $B C S S$, breast cancer-specific survival; OBC, occult breast cancer; OS, overall survival. 

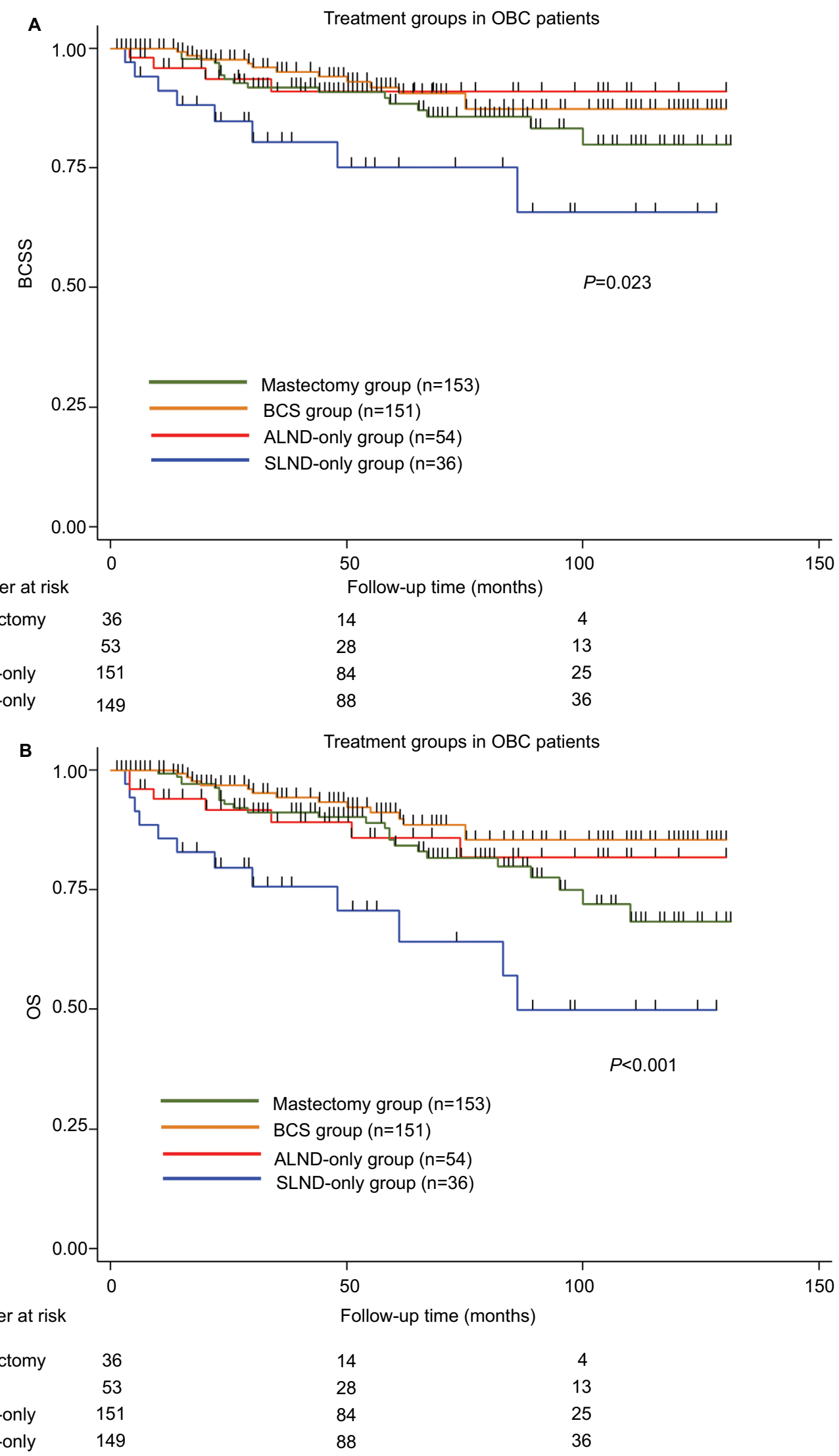

Figure 2 Kaplan-Meier survival curves according to the four groups based on different treatment strategies in OBC patients.

Notes: (A) BCSS. (B) OS. Log-rank tests were compared between groups.

Abbreviations: ALND, axillary lymph node dissection; BCS, breast-conserving surgery; BCSS, breast cancer-specific survival; OBC, occult breast cancer; OS, overall survival; SLND, sentinel lymph node dissection. 
weaknesses. Second, the incidence of locoregional recurrence is not captured in the SEER data; this omission limits our survival analysis of OBC patients. Third, records of adjuvant systemic therapy such as endocrine therapy, targeted therapy, and chemotherapy are not included in the SEER database. It is widely accepted that systemic therapy is commonly used in patients with node-positive breast cancer. ${ }^{5,42}$ These missing data, therefore, may weaken our conclusion. Large, population-based, multi-institutional analyses should be conducted to further validate these findings.

\section{Conclusion}

Our study reveals that $\mathrm{OBC}$ has unique clinicopathological characteristics compared with non-OBC. Although OBC patients showed a prognosis similar to that of the overall nonOBC population, their survival was significantly increased after adjustment for confounders. We did not observe a difference in outcomes among the mastectomy group, the BCS group, and the ALND-only group. However, there is insufficient evidence to support ALND alone as an effective treatment strategy. A thorough biological and clinical understanding of OBC might improve the clinical management of and the outcomes of this rare type of breast cancer.

\section{Acknowledgments}

This work was supported by grants from the National Natural Science Foundation of China (81502278, 81572583, 81372848, 81370075), the Training Plan of Excellent Talents in the Shanghai Municipality Health System (2017YQ038), the Training Plan of Excellent Talents of Fudan University Shanghai Cancer Center (YJYQ201602), the Municipal Project for Developing Emerging and Frontier Technology in Shanghai Hospitals (SHDC12010116), the Cooperation Project of Conquering Major Diseases in the Shanghai Municipality Health System (2013ZYJB0302), the Innovation Team of the Ministry of Education (IRT1223), and the Shanghai Key Laboratory of Breast Cancer (12DZ2260100). The funders had no role in the study design, data collection and analysis, decision to publish, or preparation of the manuscript.

\section{Author contributions}

Y-ZJ and G-HD designed the experiments. L-PG and X-YL performed most of the experiments, analyzed the data, and wrote the manuscript. Y-ZJ, YX, Z-CG, and SZ revised the manuscript. All authors contributed toward data analysis, drafting and revising the paper and agree to be accountable for all aspects of the work.

\section{Disclosure}

The authors report no conflicts of interest in this work.

\section{References}

1. Patel J, Nemoto T, Rosner D, Dao TL, Pickren JW. Axillary lymph node metastasis from an occult breast cancer. Cancer. 1981;47(12): 2923-2927.

2. Rosen PP. Axillary lymph node metastases in patients with occult noninvasive breast carcinoma. Cancer. 1980;46(5):1298-1306.

3. Owen HW, Dockerty MB, Gray HK. Occult carcinoma of the breast. Surg Gynecol Obstet. 1954;98(3):302-308.

4. Pavlidis N, Pentheroudakis G. Cancer of unknown primary site. Lancet. 2012;379(9824):1428-1435.

5. Pentheroudakis G, Lazaridis G, Pavlidis N. Axillary nodal metastases from carcinoma of unknown primary (CUPAx): a systematic review of published evidence. Breast Cancer Res Treat. 2010;119(1): $1-11$.

6. Sohn G, Son BH, Lee SJ, et al. Treatment and survival of patients with occult breast cancer with axillary lymph node metastasis: a nationwide retrospective study. J Surg Oncol. 2014;110(3):270-274.

7. Walker GV, Smith GL, Perkins GH, et al. Population-based analysis of occult primary breast cancer with axillary lymph node metastasis. Cancer. 2010;116(17):4000-4006.

8. He M, Tang LC, Yu KD, Kd Y, et al. Treatment outcomes and unfavorable prognostic factors in patients with occult breast cancer. Eur J Surg Oncol. 2012;38(11):1022-1028.

9. Rosen PP, Kimmel M. Occult breast carcinoma presenting with axillary lymph node metastases: a follow-up study of 48 patients. Hum Pathol. 1990;21(5):518-523.

10. Montagna E, Bagnardi V, Rotmensz N, et al. Immunohistochemically defined subtypes and outcome in occult breast carcinoma with axillary presentation. Breast Cancer Res Treat. 2011;129(3):867-875.

11. Ping S, Ming WH, Bin SH, et al. Comparison of clinical characteristics between occult and non-occult breast cancer. $J$ Buon. 2014;19(3):662-666.

12. Jackson B, Scott-Conner C, Moulder J. Axillary metastasis from occult breast carcinoma: diagnosis and management. Am Surg. 1995;61(5): 431-434.

13. Svastics E, Rónay P, Bodó M. Occult breast cancer presenting with axillary metastasis. Eur J Surg Oncol. 1993;19(6):575-580.

14. National Comprehensive Cancer Network (NCCN). Clinical Practice Guidelines in Oncology, Breast Cancer v3.2017; 2017.

15. Blanchard DK, Shetty PB, Hilsenbeck SG, Elledge RM. Association of surgery with improved survival in stage IV breast cancer patients. Ann Surg. 2008;247(5):732-738.

16. Olson JA, Morris EA, van Zee KJ, Linehan DC, Borgen PI. Magnetic resonance imaging facilitates breast conservation for occult breast cancer. Ann Surg Oncol. 2000;7(6):411-415.

17. Varadarajan R, Edge SB, Yu J, Watroba N, Janarthanan BR. Prognosis of occult breast carcinoma presenting as isolated axillary nodal metastasis. Oncology. 2006;71(5-6):456-459.

18. Stephen BE, Byrd DR, Michael A, editors M. AJCC Cancer Staging Manual. 7th ed. New York: Springer; 2010.

19. Austin PC. Statistical criteria for selecting the optimal number of untreated subjects matched to each treated subject when using many-to-one matching on the propensity score. Am J Epidemiol. 2010;172(9):1092-1097.

20. Hessler LK, Molitoris JK, Rosenblatt PY, et al. Factors Influencing Management and Outcome in Patients with Occult Breast Cancer with Axillary Lymph Node Involvement: Analysis of the National Cancer Database. Ann Surg Oncol. 2017;24(10):2907-2914.

21. Amir E, Miller N, Geddie W, et al. Prospective study evaluating the impact of tissue confirmation of metastatic disease in patients with breast cancer. J Clin Oncol. 2012;30(6):587-592. 
22. Lindström LS, Karlsson E, Wilking UM, et al. Clinically used breast cancer markers such as estrogen receptor, progesterone receptor, and human epidermal growth factor receptor 2 are unstable throughout tumor progression. J Clin Oncol. 2012;30(21):2601-2608.

23. Pusztai L, Viale G, Kelly CM, Hudis CA, Estrogen HCA. Estrogen and HER-2 receptor discordance between primary breast cancer and metastasis. Oncologist. 2010;15(11):1164-1168.

24. Khasraw M, Brogi E, Seidman AD. The need to examine metastatic tissue at the time of progression of breast cancer: is re-biopsy a necessity or a luxury? Curr Oncol Rep. 2011;13(1):17-25.

25. Curtit E, Nerich V, Mansi L, et al. Discordances in estrogen receptor status, progesterone receptor status, and HER2 status between primary breast cancer and metastasis. Oncologist. 2013;18(6):667-674.

26. Pentheroudakis G, Briasoulis E, Pavlidis N. Cancer of unknown primary site: missing primary or missing biology? Oncologist. 2007;12(4): 418-425.

27. Pavlidis N, Briasoulis E, Hainsworth J, Greco FA. Diagnostic and therapeutic management of cancer of an unknown primary. Eur J Cancer. 2003;39(14):1990-2005.

28. Halsted WS. I. The Results of Radical Operations for the Cure of Carcinoma of the Breast. Ann Surg. 1907;46(1):1-19.

29. Wang X, Zhao Y, Cao X. Clinical benefits of mastectomy on treatment of occult breast carcinoma presenting axillary metastases. Breast $J$. 2010;16(1):32-37

30. Merson M, Andreola S, Galimberti V, Bufalino R, Marchini S, Veronesi U. Breast carcinoma presenting as axillary metastases without evidence of a primary tumor. Cancer. 1992;70(2):504-508.

31. Abbruzzese JL, Abbruzzese MC, Lenzi R, Hess KR, Raber MN. Analysis of a diagnostic strategy for patients with suspected tumors of unknown origin. J Clin Oncol. 1995;13(8):2094-2103.

32. Kemeny MM, Rivera DE, Terz JJ, Benfield JR. Occult primary adenocarcinoma with axillary metastases. Am J Surg. 1986;152(1) 43-47.
33. Barton SR, Smith IE, Kirby AM, Ashley S, Walsh G, Parton M. The role of ipsilateral breast radiotherapy in management of occult primary breast cancer presenting as axillary lymphadenopathy. Eur J Cancer. 2011;47(14):2099-2106.

34. Shannon C, Walsh G, Sapunar F, A'Hern R, Smith I. Occult primary breast carcinoma presenting as axillary lymphadenopathy. Breast. 2002;11(5):414-418.

35. Foroudi F, Tiver KW. Occult breast carcinoma presenting as axillary metastases. Int J Radiat Oncol Biol Phys. 2000;47(1):143-147.

36. Blanchard DK, Farley DR. Retrospective study of women presenting with axillary metastases from occult breast carcinoma. World J Surg. 2004;28(6):535-539.

37. Rueth NM, Black DM, Limmer AR, et al. Breast conservation in the setting of contemporary multimodality treatment provides excellent outcomes for patients with occult primary breast cancer. Ann Surg Oncol. 2015;22(1):90-95.

38. Campana F, Fourquet A, Ashby MA, et al. Presentation of axillary lymphadenopathy without detectable breast primary (T0 N1b breast cancer): experience at Institut Curie. Radiother Oncol. 1989;15(4):321-325.

39. Ellerbroek N, Holmes F, Singletary E, Evans H, Oswald M, Mcneese $\mathrm{M}$. Treatment of patients with isolated axillary nodal metastases from an occult primary carcinoma consistent with breast origin. Cancer. 1990;66(7):1461-1467.

40. Kim BH, Kwon J, Kim K. Evaluation of the Benefit of Radiotherapy in Patients with Occult Breast Cancer: A Population-Based Analysis of the SEER Database. Cancer Res Treat. 2018;50(2):551-561.

41. Macedo FI, Eid JJ, Flynn J, Jacobs MJ, Mittal VK. Optimal Surgical Management for Occult Breast Carcinoma: A Meta-analysis. Ann Surg Oncol. 2016;23(6):1838-1844.

42. Curigliano G, Burstein HJ, P Winer E, Pw E, et al. De-escalating and escalating treatments for early-stage breast cancer: the St. Gallen International Expert Consensus Conference on the Primary Therapy of Early Breast Cancer 2017. Ann Oncol. 2017;28(8):1700-1712.
Cancer Management and Research

\section{Publish your work in this journal}

Cancer Management and Research is an international, peer-reviewed open access journal focusing on cancer research and the optimal use of preventative and integrated treatment interventions to achieve improved outcomes, enhanced survival and quality of life for the cancer patient The manuscript management system is completely online and includes

\section{Dovepress}

a very quick and fair peer-review system, which is all easy to use. Visit http://www.dovepress.com/testimonials.php to read real quotes from published authors. 